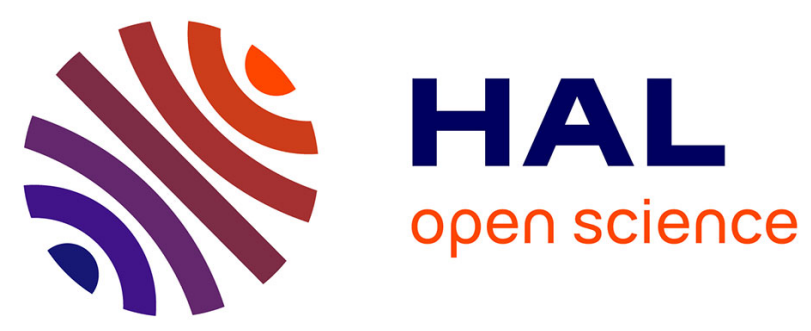

\title{
What is the depth of field reachable in practice with generic binary phase masks and digital deconvolution?
}

Alice Fontbonne, Hervé Sauer, Caroline Kulcsár, Anne-Lise Coutrot, François

Goudail

\section{- To cite this version:}

Alice Fontbonne, Hervé Sauer, Caroline Kulcsár, Anne-Lise Coutrot, François Goudail. What is the depth of field reachable in practice with generic binary phase masks and digital deconvolution?. Unconventional Optical Imaging II, Apr 2020, Online Only, France. pp.14, 10.1117/12.2557731. hal-02529673

\section{HAL Id: hal-02529673 \\ https://hal.science/hal-02529673}

Submitted on 2 Apr 2020

HAL is a multi-disciplinary open access archive for the deposit and dissemination of scientific research documents, whether they are published or not. The documents may come from teaching and research institutions in France or abroad, or from public or private research centers.
L'archive ouverte pluridisciplinaire HAL, est destinée au dépôt et à la diffusion de documents scientifiques de niveau recherche, publiés ou non, émanant des établissements d'enseignement et de recherche français ou étrangers, des laboratoires publics ou privés. 


\title{
What is the depth of field reachable in practice with generic binary phase masks and digital deconvolution?
}

\author{
Alice Fontbonne*a, Hervé Sauer ${ }^{\mathrm{a}}$, Caroline Kulcsár ${ }^{\mathrm{a}}$, Anne-Lise Coutrot ${ }^{\mathrm{a}}$, François Goudail ${ }^{\mathrm{a}}$ \\ a'Université Paris-Saclay, Institut d'Optique Graduate School, CNRS, Laboratoire Charles Fabry, \\ 91120, Palaiseau, France
}

\begin{abstract}
We investigate the practical behavior of a co-optimized hybrid system involving a generic binary phase mask and digital deconvolution. We perform experiments with a case-study optical system with observed scene lighting by LED of different colors. By imaging a real scene and a depth of field (DoF) target, we show that the DoF reachable in practice matches with good accuracy the one predicted by simulation in case of monochromatic illumination. We also characterize the drop in performance when using this type of system with actual illumination wavelength departing from the nominal one.
\end{abstract}

Keywords: image processing; binary phase masks; deconvolution; optical system codesign; wavefront coding; experimental validation.

This pre-print has been published as: Alice Fontbonne, Hervé Sauer, Caroline Kulcsár, Anne-Lise Coutrot, and François Goudail "What is the depth of field reachable in practice with generic binary phase masks and digital deconvolution?", Proc. SPIE 11351, Unconventional Optical Imaging II, 113510F (30 March 2020);

DOI: https://doi.org/10.1117/12.2557731

\section{INTRODUCTION}

Generic optical systems with a given aperture have limited depth of field (DoF). It is well known that this DoF can be extended by adding a co-designed phase mask in the pupil of the lens [1] [2] [3]. Since adding a phase mask tends to decrease the raw image quality, it is possible to perform digital image processing after acquisition to regain optimal quality [4] [5] [6]. In this paper, we study the DoF reachable in practice by generic binary phase masks associated with digital deconvolution based on the average Wiener filter [7]. Their performance has already been demonstrated by simulation [8] and we have reported a first experimental study under narrow-band illumination [9].

In the present paper, we investigate the DoF reachable in practice with such a hybrid imaging system when scene illumination, albeit monochromatic, has a wavelength different from that for which the system has been optimized. For that purpose, we characterize their through-focus behavior by simulation and by imaging a DoF target and a real scene.

This article is organized as follows: firstly, we expose the binary phase mask co-optimization method and describe the case-study optical system we have designed to evaluate the phase masks (Section 2). Secondly, we present simulated and experimental characterization of the system through-focus behavior using DoF target images (Section 3). Thirdly, we analyze a real scene image in order to check the visual quality of the images obtained with the hybrid system (Section 4). Finally, we conclude in Section 5 about the DoF reachable in practice with this type of systems. 


\section{METHODOLOGY}

\subsection{Binary phase masks optimization}

We consider hybrid optical-digital hybrid systems composed of a unique co-optimized optical element, a binary annular phase mask, consisting of a series of $N$ concentric annular regions of phase modulation of alternatively 0 or $\pi$ rad at the nominal wavelength $\lambda_{0}=625 \mathrm{~nm}$ (see Figure 1). Each annular constant-phase area corresponds to a "ring" and the mask is parameterized by the values of the $N-1$ normalized outer radii $\phi=\left\{\rho_{1}, \ldots, \rho_{N-1}\right\}$, where $\phi$ satisfies the condition $0<$ $\rho_{1}<\cdots<\rho_{N-1}<\rho_{N}=1$.

The goal is to extend the DoF of the optical system. During the optimization of the phase mask, we therefore consider objects at different defocus positions. The defocus parameter is defined as

$$
\psi\left(\Delta z_{o}\right)=\frac{\left(z_{i} \times N A\right)^{2}}{2}\left(\frac{1}{z_{i}}+\frac{1}{z_{o 0}+\Delta z_{o}}-\frac{1}{f_{\lambda_{0}}}\right)
$$

where $z_{o 0}$ is the distance of a well-focused object to the imaging system, $z_{o}=z_{o 0}+\Delta z_{o}$ is the distance of a defocused object , NA is the image numerical aperture, $z_{i}$ is the position of the sensor and $f_{\lambda_{0}}$ is the focal length of the optical system at wavelength $\lambda_{0}$.

The image produced on the sensor can be modeled by $h_{\psi}^{\phi}(r) * O(r)$ where $h_{\psi}^{\phi}(r)$ is the point spread function (PSF) of the optical system (depending on the defocus parameter $\psi$ and on the radius values of the phase mask $\phi$ ), $*$ denotes the convolution operator and $O(r)$ is the ideal sampled scene image ( $r$ represents the spatial coordinates). The acquired raw image is then restored with the averaged Wiener filter $w^{\phi}(r)$ [7]. The output of the hybrid optical-digital system is modeled by $\hat{O}(r)=w^{\phi}(r) *\left[h_{\psi}^{\phi}(r) * O(r)+n(r)\right]$ where $n(r)$ is the detection noise.

For each mask, we choose the number of rings $N$ to optimize and a targeted defocus range $\psi_{\max }$, which corresponds to the maximal value of the defocus parameter. With the "particule swarm" optimization algorithm [10], we determine the phase mask and the deconvolution filter $w^{\phi}(r)$ that jointly minimize the mean-squared error between the ideal image $O(r)$ and the reconstructed one $\hat{O}(r)[8]$.

We consider here the optimal masks (within the meaning of the above mentioned MSE criterion) with the fewest rings, for three different targeted defocus range parameters $\psi_{\max }$. The optimal mask for $\psi_{\max }=1 \lambda_{0}$ is denoted " $1 \lambda_{0}$-mask". We denote " $2 \lambda_{0}$-mask" the phase mask for $\psi_{\max }=2 \lambda_{0}$ and " $2.5 \lambda_{0}$-mask" the phase mask for $\psi_{\max }=2.5 \lambda_{0}$ [8]. The goal of this article is to evaluate the behavior of these masks when they are integrated in a near perfect, case-study optical system, and to check out their robustness to illumination wavelength.

(a)

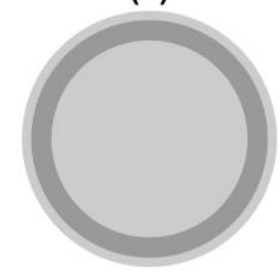

(b)

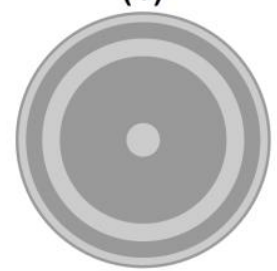

(c)

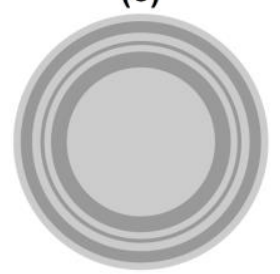

Figure 1. Three examples of binary phase masks. Dark gray areas induce a phase shift of 0 and light gray areas induce a phase shift of $\pi$ rad at the nominal wavelength $\lambda_{0}$. Those three phase masks are called (a) $1 \lambda_{0}$-mask (b) $2 \lambda_{0}$-mask and (c) $2.5 \lambda_{0^{-}}$ mask (see explanations in the text).

\subsection{Case-study optical system}

For that purpose, we design a case-study optical system made of two commercial doublets [see elements (a) and (d) on Figure 2] and a CMOS camera sensor [element (e)], with the following characteristics: 
- $\quad$ The system has an image $F$-number of 12.5 corresponding to a cutoff image spatial frequency of the system at $\lambda_{0}=625 \mathrm{~nm}$ of $128 \mathrm{lp} \cdot \mathrm{mm}^{-1}$. With a $3.45-\mu \mathrm{m}$ pitch CMOS camera sensor, the Nyquist frequency is $145 \mathrm{lp} . \mathrm{mm}^{-1}$ and the system PSF is thus correctly sampled at $\lambda_{0}$.

- The magnification of the system is -1.00 and independent of the axial position thanks to enforced object telecentricity of the designed optical system. The object and image field-of-view is $8.5 \mathrm{~mm} \times 7.09 \mathrm{~mm}$.

- $\quad$ The removable phase mask [element (c)] is placed as close as possible to the STOP [element (b)]. It has a clear aperture diameter of $\varnothing 20 \mathrm{~mm}$. An aberration plate can be added next to it to control phase mask robustness to the chosen aberration [element $(\mathrm{g})]$.

- The scene is illuminated with a LED lighting system [element (h)]. The $1 \lambda_{0}$-mask, $2 \lambda_{0}$-mask and $2.5 \lambda_{0}$-mask are optimal for a red LED illumination having a narrow spectrum centered at $625 \mathrm{~nm}$ and a full-width at halfmaximum of $20 \mathrm{~nm}$. We can also use the blue LED or the green LED, which have the spectrum displayed on Figure 3, in order to evaluate the robustness of the masks when the illumination spectrum is different from the nominal one. In order to avoid any stray-light concern, the optical components are set in place by an optomechanical closed housing [element (h)].

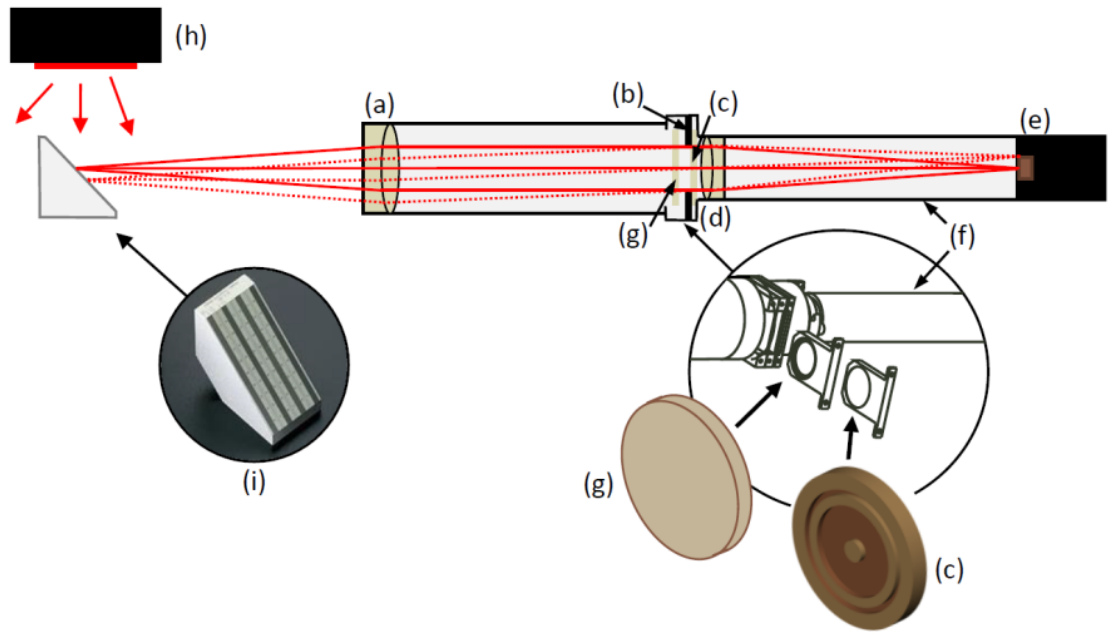

Figure 2. Schematic drawing of the optical system designed for the study of the phase masks: (a) first commercial doublet, (b) STOP, (c) removable phase mask, (d) second commercial doublet, (e) camera sensor, (f) optomechanical closed housing, (g) optional removable aberration plate, (h) LED lighting system, and (i) scene, for instance, DoF target.

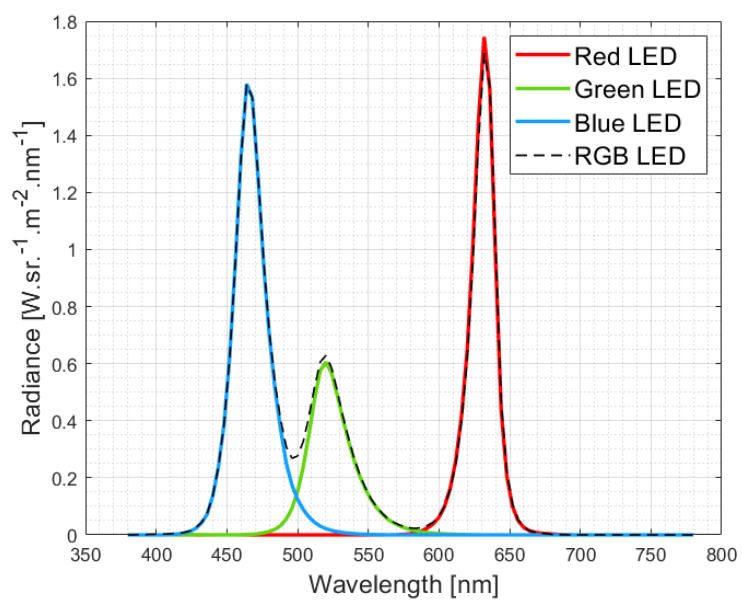

Figure 3. Spectrum of the LED. Dominant wavelengths are $\lambda_{B}=470 \mathrm{~nm}, \lambda_{G}=528 \mathrm{~nm}$ and $\lambda_{0}=625 \mathrm{~nm}$. 


\section{THROUGH-FOCUS BEHAVIOR}

Let us first characterize the through-focus behavior of the phase masks. Firstly, we study by simulation the mask behaviors at three different illumination wavelengths. Secondly, we experimentally check out the results with images of a DoF target.

\subsection{Simulated through-focus behavior of phase masks}

In order to have an insight of the DoF of the hybrid system it is useful to compute the contrast transfer function (CTF) of the optical part of the hybrid system as object defocus distance varies. The CTF is the local contrast $\frac{\max -\min }{\max +\min }$ of the image of a perfect Ronchi ruling pattern. It can be done with $\mathrm{CodeV}^{\odot}$ by computing the modulation transfer function (MTF) with a square wave (the SQW option). The distance between the two first nullings of the CTF can be used as a rough estimation of the DoF range at a given spatial frequency.

For the optical system without phase mask, we observe in Figure 4 the almost perfectly symmetrical behavior of the optical system illuminated with wavelength $\lambda_{0}$ at spatial frequencies 15 lp.mm $\mathrm{mm}^{-1}$ (Figure 4(a)) and 40 lp.mm ${ }^{-1}$ (Figure 4(b)). At $15 \mathrm{lp} . \mathrm{mm}^{-1}$, the first nullings occur at object defocus distances $-1.25 \mathrm{~mm}$ and $1.25 \mathrm{~mm}$. At $40 \mathrm{lp} . \mathrm{mm}^{-1}$, as the MTF is lower for higher frequencies for a perfect optical system, the first nullings appear sooner, at $-0.5 \mathrm{~mm}$ and $0.5 \mathrm{~mm}$. We observe in both cases that the optical system presents a folded axial chromatic aberration: both red and blue lights are focused at a greater distance than the green one. For the three wavelengths, the distance between the two first CTF nullings is the same, but the heights of the peaks are different. For the spatial frequency $15 \mathrm{lp} . \mathrm{mm}^{-1}$, as for the spatial frequency $40 \mathrm{lp} . \mathrm{mm}^{-1}$, the maximal value of the CTF is higher for the blue wavelength, than for the green wavelength, or for the red wavelength. This was predictable since when the wavelength is short, the MTF of a diffraction limited optical system presents higher levels for a given spatial frequency.

(a)

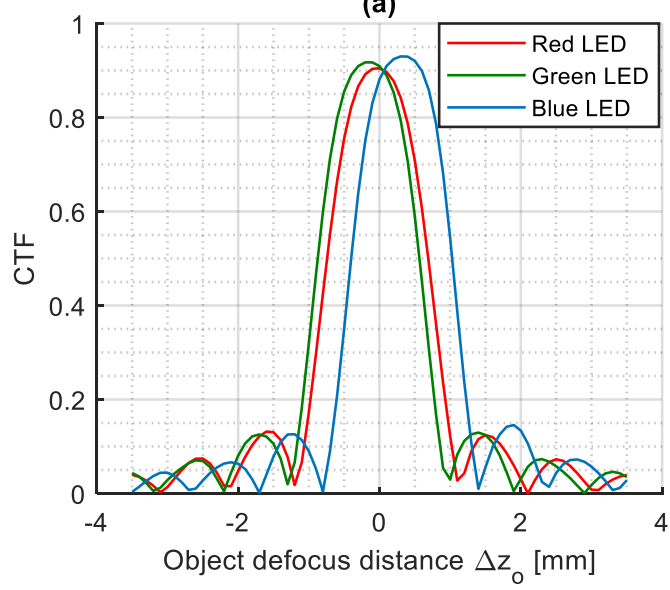

(b)

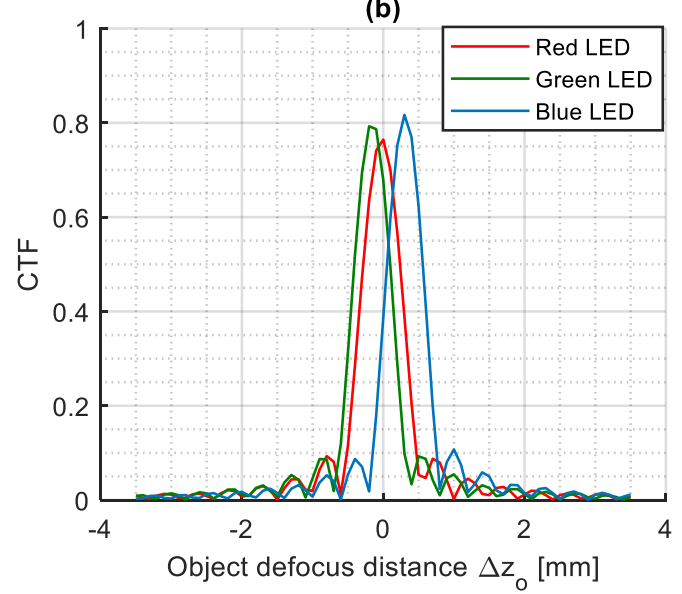

Figure 4. CTF of the optical system without phase mask for the three wavelengths $\lambda_{B}=470 \mathrm{~nm}, \lambda_{G}=528 \mathrm{~nm}$ and $\lambda_{0}=$ $625 \mathrm{~nm}$ observed at (a) $15 \mathrm{lp} \cdot \mathrm{mm}^{-1}$ and (b) $40 \mathrm{lp} \cdot \mathrm{mm}^{-1}$.

When a phase mask is integrated in the optical system, it changes its through-focus behavior. Let us first consider the red wavelength $\lambda_{0}$ for which the phase masks have been optimized. It is seen that the distance between the two first nullings increases with the targeted DoF range, both for the spatial frequency 15 lp.mm-1 (Figure 5, first column) and 40 lp.mm $\mathrm{mm}^{-1}$ (Figure 5, second column). At the spatial frequency $15 \mathrm{lp} . \mathrm{mm}^{-1}$, this distance is equal to $4 \mathrm{~mm}$ with the $1 \lambda_{0}$-mask (Figure 5(a)), $5.2 \mathrm{~mm}$ with de $2 \lambda_{0}$-mask (Figure 5(c)), and $5.8 \mathrm{~mm}$ with the $2.5 \lambda_{0}$-mask (Figure 5(e)). At the spatial frequency $40 \mathrm{lp} . \mathrm{mm}^{-1}$, the distance between two nullings is equal to $2.4 \mathrm{~mm}$ with the $1 \lambda_{0}$-mask (Figure 5(b)), $3.6 \mathrm{~mm}$ with the $2 \lambda_{0}$ mask (Figure 5(d)), and $4.2 \mathrm{~mm}$ with the $2.5 \lambda_{0}$-mask (Figure 5(f)). With these simulations at the nominal wavelength $\lambda_{0}$, we can therefore conclude that the distance between the two first nullings increases with the targeted DoF range.

The CTF simulation also allows us to verify that the phase masks actually increase the DoF range as targeted. Indeed, with the considered optical system, the $1 \lambda_{0}$-mask should increase the DoF up to an object defocus range of $\pm 0.8 \mathrm{~mm}$, the $2 \lambda_{0}$ - 
mask to $\pm 1.6 \mathrm{~mm}$, and the $2.5 \lambda_{0}$-mask to $\pm 2 \mathrm{~mm}$ (see Eq. (1) and Ref. [9]). In order the final image to be sharp and devoid of artifact after deconvolution, the CTF should be constant and large enough within this range. For example, for the $1 \lambda_{0}-$ mask at $15 \mathrm{lp} . \mathrm{mm}^{-1}$ (Figure 5(a)), it is approximately flat between the maxima of the CTF located at $\pm 0.8 \mathrm{~mm}$, which is exactly the targeted DOF range. However, the actual DoF range at this frequency is larger. If we define it as the set of defocus values for which the value of the CTF is higher than 0.36 (i.e. the value of the CTF on focus), one obtains the range $\pm 1.3 \mathrm{~mm}$. For the larger spatial frequency $40 \mathrm{lp} . \mathrm{mm}^{-1}$, it is seen in Figure 5(b) that the CTF is less flat but remains significant within the targeted DoF range.

If we now consider the green wavelength $\lambda_{G}$, we notice that the distances between the two first nullings of the CTFs (for all the masks and the two spatial frequencies) are similar to those observed for the nominal red wavelength $\lambda_{0}$. However, even if the CTF values are larger than with the nominal wavelength, their variation with DoF is much less flat and clearly asymmetric, which is likely to incur to artifacts after deconvolution. 
(a)

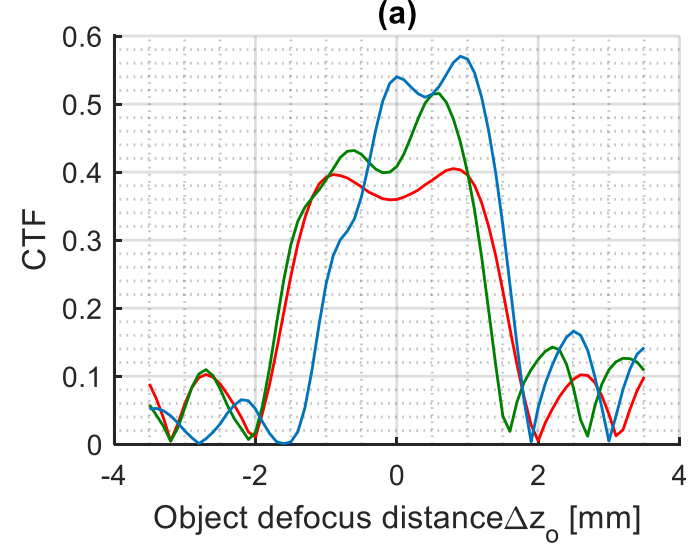

(c)

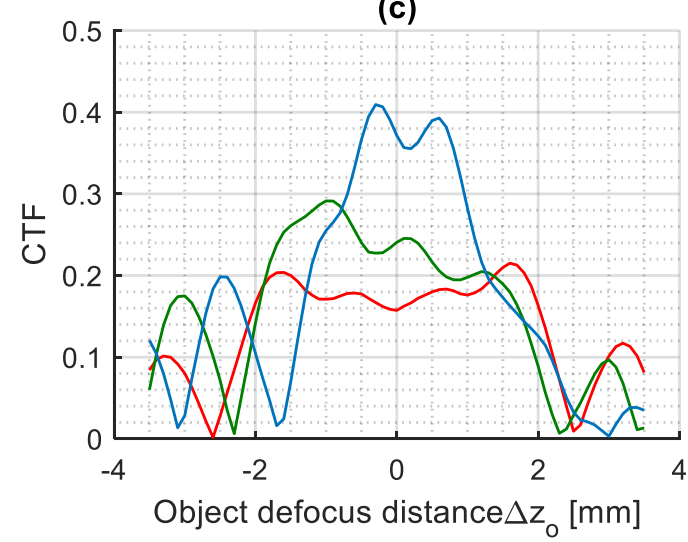

(e)

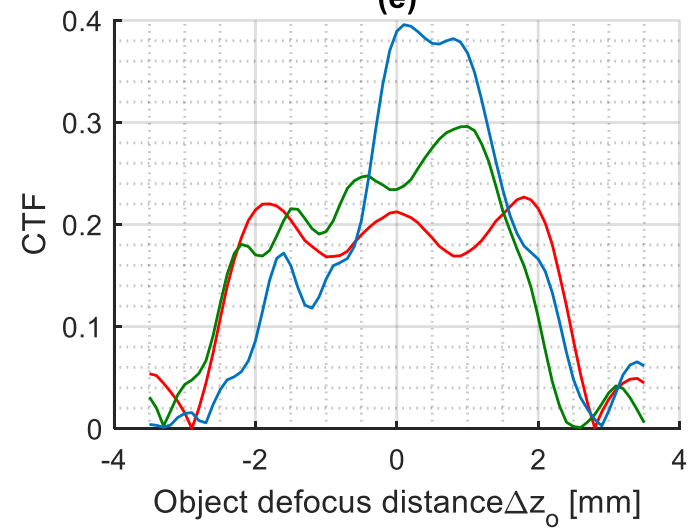

(b)

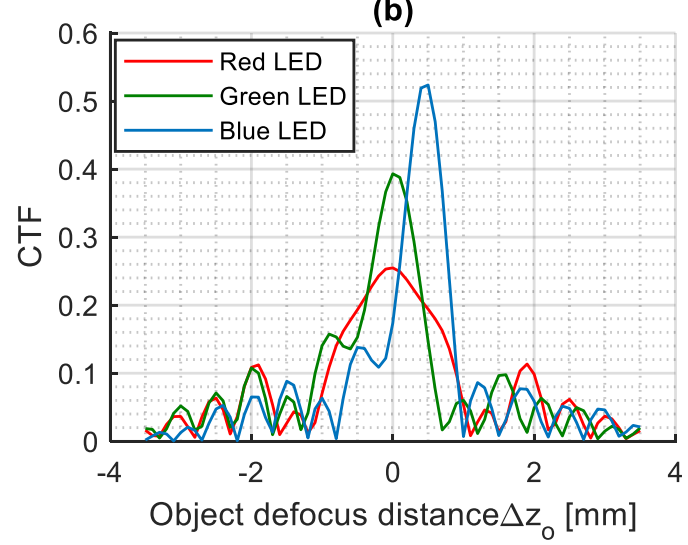

(d)

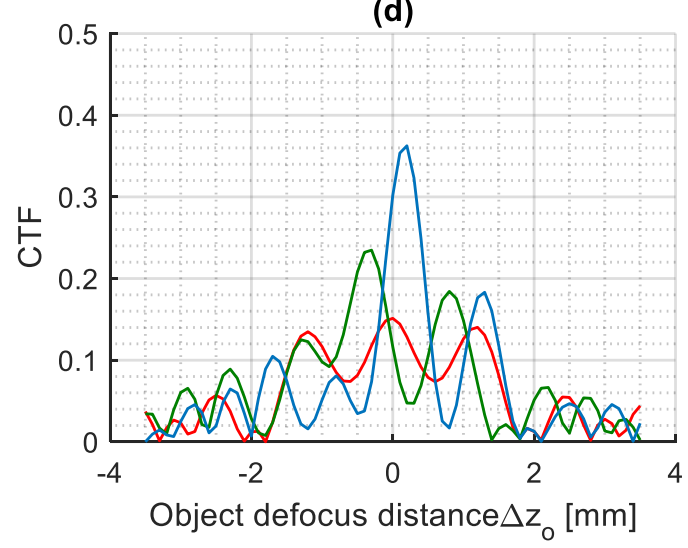

(f)

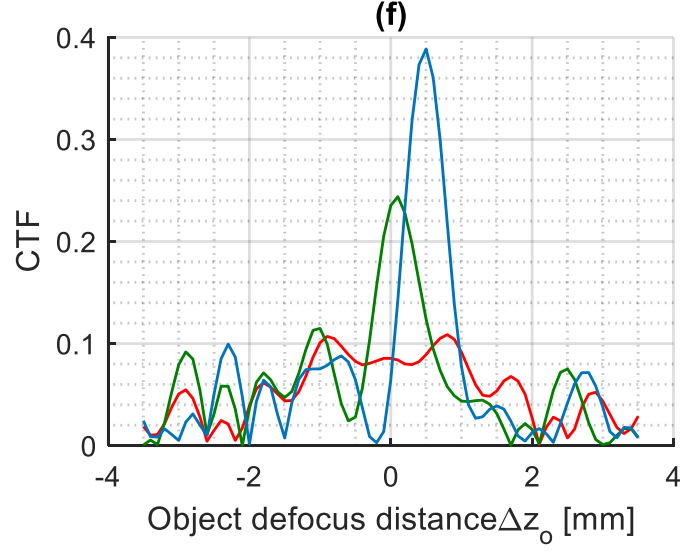

Figure 5. CTF of the optical system containing the (a-b) $1 \lambda_{0}$-mask, (c-d) $2 \lambda_{0}$-mask and (e-f) $2.5 \lambda_{0}$-mask for the three wavelengths $\lambda_{B}=470 \mathrm{~nm}, \lambda_{G}=528 \mathrm{~nm}$ and $\lambda_{0}=625 \mathrm{~nm}$ (the legend is the same as (b) for all graphs). The CTF are observed at $15 \mathrm{lp} \cdot \mathrm{mm}^{-1}$ (first column) and $40 \mathrm{lp} \cdot \mathrm{mm}^{-1}$ (second column).

This problem is even more pronounced as the used wavelength gets farther from the nominal one. At the blue wavelength $\lambda_{B}$, the behavior through focus is extremely asymmetric. The DoF range for which the CTF is flat is narrower. For example, 
for the $2.5 \lambda_{0}$-mask at $15 \mathrm{lp} . \mathrm{mm}^{-1}$ (Figure 5(e)), the DoF range at the end of the hybrid system is probably closer to $1.5 \mathrm{~mm}$ than to the original $4.5 \mathrm{~mm}$ at the nominal wavelength. Yet, the two first nullings are the same. The problem is equivalent at $40 \mathrm{lp} . \mathrm{mm}^{-1}$ (Figure 5(f)): at the wavelength $\lambda_{B}$, the through-focus behavior is starting to look like the one obtained without phase mask (Figure 4(b)). The DoF range is no longer increased by the mask at this spatial frequency.

As a conclusion, we first observe that the three optimized phase masks do extend the DoF range for the two chosen spatial frequencies when they are used with their nominal wavelength. As it could be somewhat expected, the DoF extension ability of the hybrid system decreases as the used wavelength goes away from the (red) nominal one. However, it is still significant in the green, even though it is poor in the blue. We will now experimentally verify these results.

\subsection{Experimental through-focus behavior of the phase masks}

In order to experimentally verify the through-focus behavior of the hybrid imaging system, we use a DoF target (Edmund Optics \#54-440), which is made of a Ronchi ruling test pattern drawn on a surface set at $45^{\circ}$ of the optical axis (see Figure 2(i)). When it is observed with an object-telecentric optical system, the imaged spatial frequency is constant, equal to 15 lp. $\mathrm{mm}^{-1}$, and the axial distance varies linearly with the position in the field-of-view. The images of this DoF target obtained by the imaging system can be compared with the simulated CTFs.

We represent in Figure 6 the images obtained with the optical system without phase mask (Figure 6(a)) and the images obtained with the hybrid systems involving the phase masks and digital deconvolution (Figure 6(b-c-d)), when the illumination is monochromatic at the nominal wavelength $\lambda_{0}$. We first notice the DoF enhancement: the larger the targeted DoF range, the larger the DoF range in which the image is well contrasted. In Figure 6(a), the contrast nullings appear at the same object defocus distance than for the simulated CTF Figure 4(a). Moreover, the DoF range over which the image is well contrasted is equal to that predicted by simulation. For the $1 \lambda_{0}$-mask (Figure $6(\mathrm{~b})$ ), the image is well contrasted between $-1.3 \mathrm{~mm}$ and $1.3 \mathrm{~mm}$, as seen in Figure 5(a). For the $2 \lambda_{0}$-mask (Figure 6(c)), the image is well contrasted between $-2 \mathrm{~mm}$ and $2 \mathrm{~mm}$, and for the $2.5 \lambda_{0}$-mask (Figure $6(\mathrm{~d})$ ), it is well contrasted between $-2.5 \mathrm{~mm}$ and $2.5 \mathrm{~mm}$. These results are very similar to those obtained by simulation in Figure 5(c) and Figure 5(e). Therefore, we can conclude that, at the chosen spatial frequency $15 \mathrm{lp} . \mathrm{mm}^{-1}$, the DoF reachable in practice with the hybrid system is extremely close to the one we simulated.

Let us now consider the two other monochromatic wavelengths of illumination, $\lambda_{G}=528 \mathrm{~nm}$ and $\lambda_{B}=470 \mathrm{~nm}$. We have represented in Figure 7 the images of the DoF target for the four configurations of the imaging system and these two illuminations. These results can be compared with those obtained at nominal wavelength in Figure 6. Firstly, the imaging system without phase mask keeps the same DoF independently of the wavelength of illumination (see Figure 7 - No phase mask (R-G-B)). The only difference is the shifted position of the maximal contrast, slightly toward negative object defocus distance for $\lambda_{G}$ and a little more strongly toward positive object defocus distance for $\lambda_{B}$. The experiments with the $1 \lambda_{0}-$ mask (see Figure $7-1 \lambda_{0}$-mask (R-G-B)) also validate the simulation on Figure 5(a). We notice for instance that the DoF range is a little narrower when the illumination is green instead of red, and it is clearly divided by two and shifted toward positive object defocus values when the illumination is blue. For the $2 \lambda_{0}$-mask, a similar comparison between experiment (see Figure $7-2 \lambda_{0}$-mask (R-G-B)) and simulation (Figure $5(\mathrm{c})$ ) is possible. 
(a)

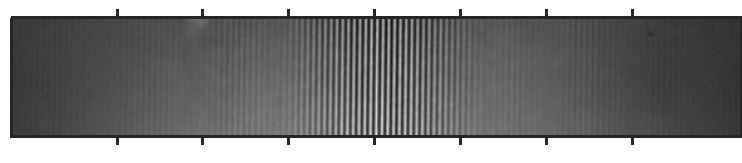

(b)

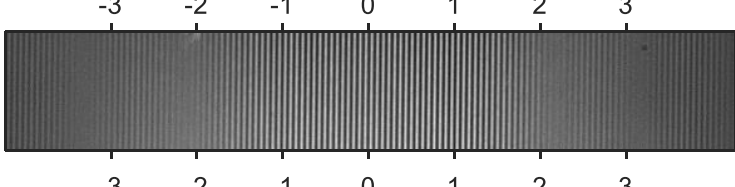

(c)

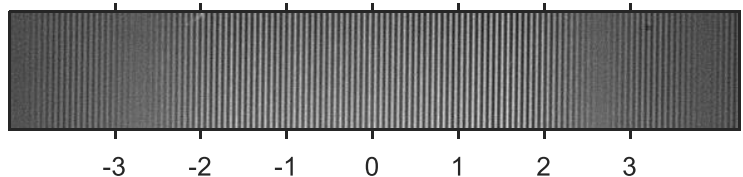

(d)

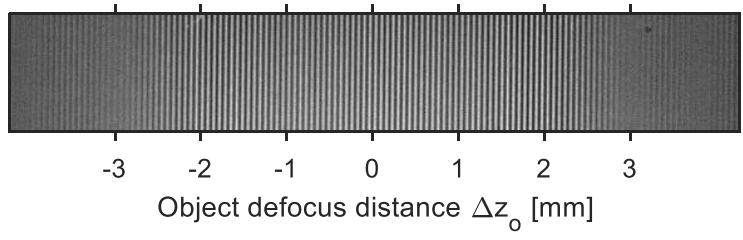

Figure 6. Images of the $15 \mathrm{lp} . \mathrm{mm}^{-1} \mathrm{DoF}$ target illuminated at the monochromatic wavelength $\lambda_{0}=625 \mathrm{~nm}$. (a) optical system without phase mask (b) hybrid system with the $1 \lambda_{0}$-mask and deconvolution (c) hybrid system with the $2 \lambda_{0}$-mask and deconvolution (d) hybrid system with the 2.5-mask and deconvolution.
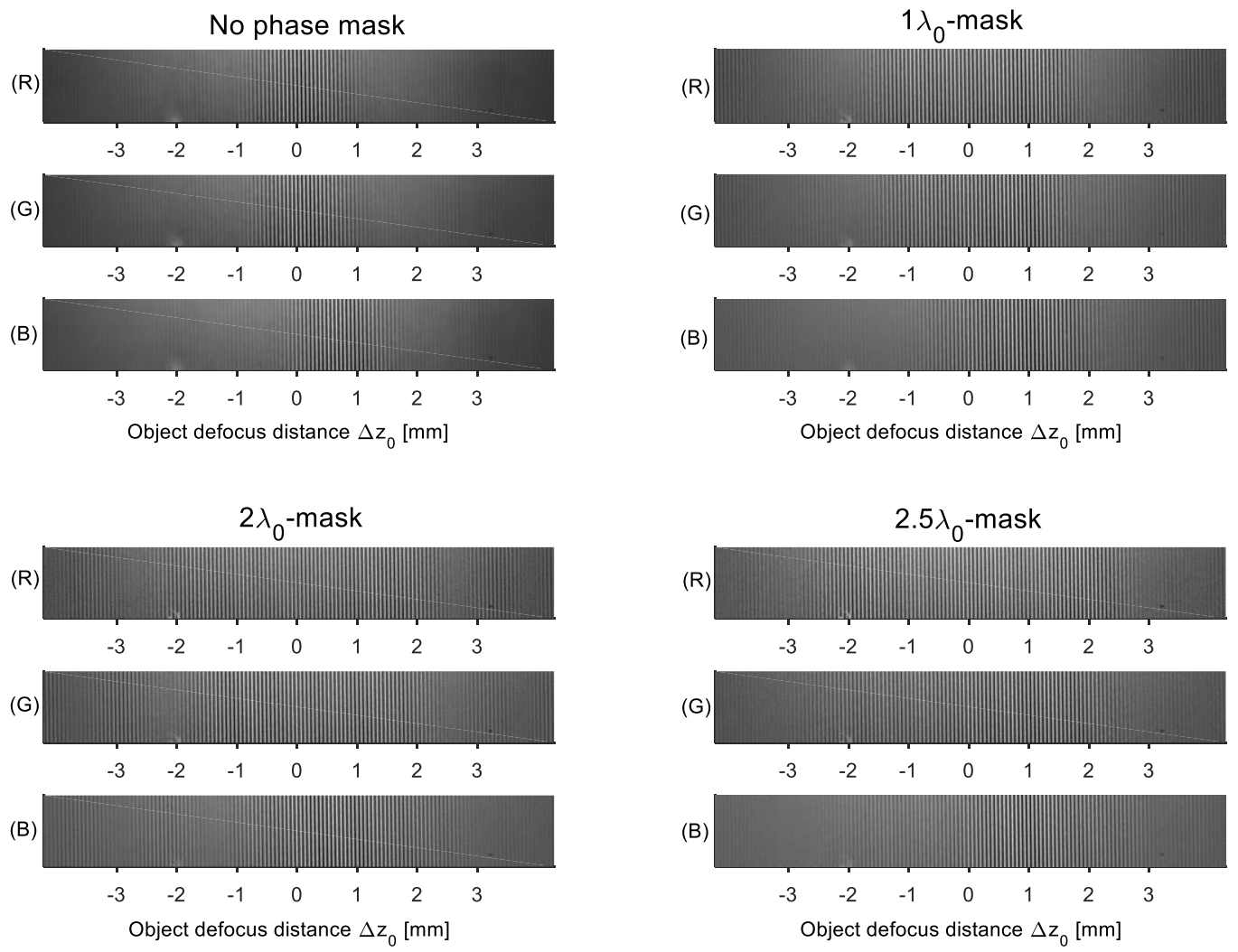

Figure 7. Images of the $15 \mathrm{lp} \cdot \mathrm{mm}^{-1} \mathrm{DoF}$ target for different imaging systems when they are illuminated at the monochromatic wavelength (R) $\lambda_{0}=625 \mathrm{~nm},(\mathrm{G}) \lambda_{G}=528 \mathrm{~nm}$ and (B) $\lambda_{B}=470 \mathrm{~nm}$. 
For the $2.5 \lambda_{0}$-mask, the experimental results (see Figure $7-2.5 \lambda_{0}$-mask (R-G-B)) validate again the simulations (Figure $5(\mathrm{e}))$. At the illumination wavelength $\lambda_{G}$, the DoF range is close to the one obtained with an illumination at $\lambda_{0}$. We can nevertheless notice that within this range, the contrast is not stable with the object defocus distance: it increases from left to right. As in the simulation, the first nulling for positive object defocus value is obtained sooner at $\lambda_{G}$ than at $\lambda_{0}$. For the illumination at $\lambda_{B}$, we observe a "two stage" contrast, with a low contrast for negative defocus values and a high contrast for positive defocus values. Moreover, the positions of the nullings are the same at wavelength $\lambda_{B}$ and wavelength $\lambda_{0}$, exactly as seen on Figure 5(e).

In conclusion, at $15 \mathrm{lp} . \mathrm{mm}^{-1}$, the experimental results are in good agreement with the simulated ones. Therefore, the phase masks optimized at the wavelength $\lambda_{0}$ are robust to their use under another monochromatic illumination only if the wavelength is close enough to $\lambda_{0}$. In the other cases, it induces a drop in the image quality over the targeted DoF range (as observed for the wavelength $\lambda_{G}$ ), or even to a drastic reduction of this range (as observed for the wavelength $\lambda_{B}$ ).

As a final element of characterization, we test the hybrid systems with a real scene (containing therefore several spatial frequencies) illuminated with these three monochromatic wavelengths.

\section{REAL SCENE IMAGES}

To analyze the differences between mask behavior at several monochromatic illuminations, we focus our study on the $2 \lambda_{0}$ mask and compare its behavior to an optical system without phase mask. The scene we image, Figure 8 , consists of a strand of wool surrounded by a chain. The focus is made between the fourth and the fifth link of the chain from the left.

(a)

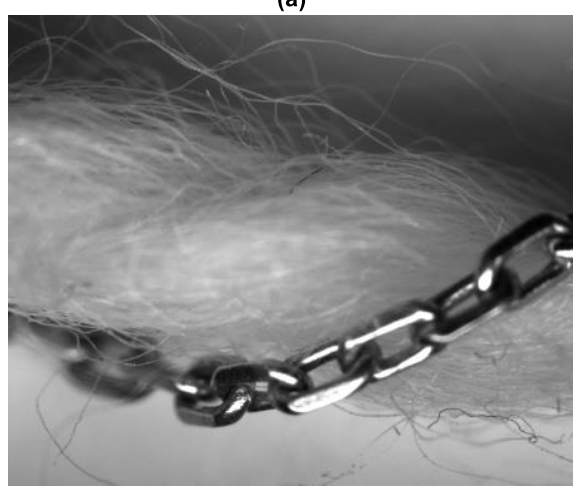

(c)

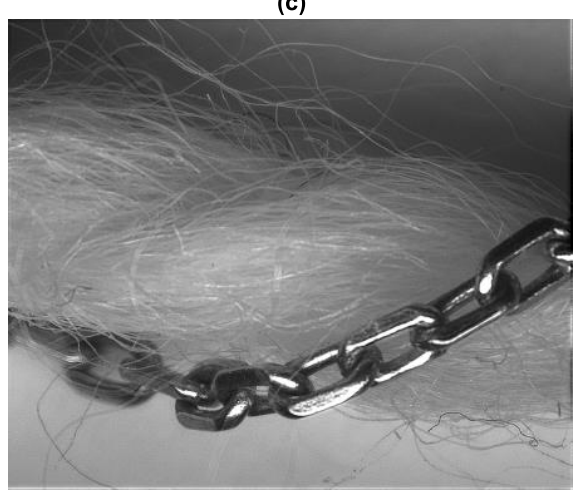

(b)

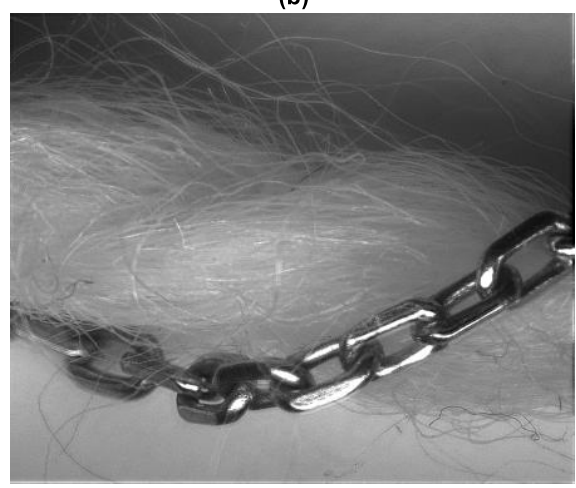

(d)

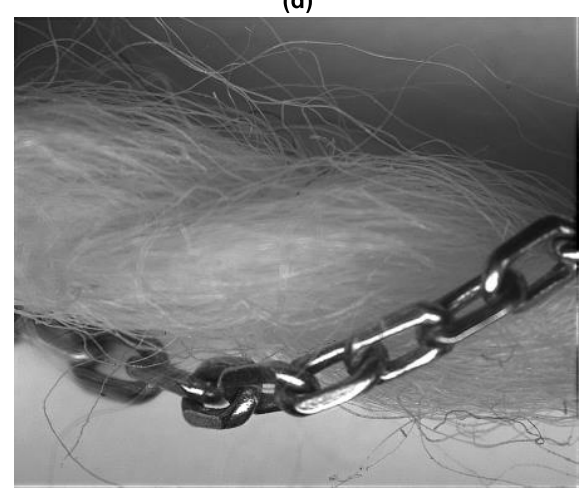

Figure 8. Image of a scene (a) with an optical system without phase mask (b) with the $2 \lambda_{0}$-mask and an illumination at $\lambda_{0}$ (c) with the $2 \lambda_{0}$-mask and an illumination at $\lambda_{G}$ (d) with the $2 \lambda_{0}$-mask and an illumination at $\lambda_{B}$. (b-c-d) are deconvolved. 
Each link of the chain is about $1 \mathrm{~mm}$ long. In the left part of the image, the links of the chain are far away from the lens (at about 3 or $4 \mathrm{~mm}$ behind the focus plane), while the links on the right are close to it ( 2 or $3 \mathrm{~mm}$ in front of the focus plane). Because of its thickness of about $2 \mathrm{~mm}$, the woolen strand is unfocused, except for its sides on the top and the bottom of the image.

In Figure 8(a), the image obtained at $\lambda_{0}$ without phase mask is sharp only at the focus position (see the on-focus links of the chain and the wool threads in top and bottom parts of the image). All the other links are blurred, the details on the metal are not visible, and we cannot distinguish the threads of the woolen strand in the center of the picture.

When the scene is observed with the $2 \lambda_{0}$-mask at $\lambda_{0}$ (Figure $8(\mathrm{~b})$ ) the DoF is clearly increased. Three supplementary links are now sharp, and the threads of the woolen strand in the center of the picture are visible. This confirms the DoF increase with a phase mask.

There is no noticeable difference with the illumination at $\lambda_{G}$ (Figure 8(c)): the DoF is still improved. This was predictable considering the simulations. We have seen in Figure 5(c-d) that the $2 \lambda_{0}$-mask used at $\lambda_{G}$ does increase the DoF but with an image quality depending on the object defocus distance. Thus, an image containing different spatial frequencies at different object defocus positions may have lower image quality than the image of the scene at $\lambda_{0}$ at some points, and better one at others. Therefore, the phase mask is robust to its use at $\lambda_{G}$, even though $\lambda_{G}$ is $77 \mathrm{~nm}(16 \%)$ lower than $\lambda_{0}$.

Nevertheless, it is not the case if the monochromatic illumination is too far from the nominal one. The drop in quality at the illumination wavelength $\lambda_{B}$ is important: we cannot see anymore the details in the center of the image. Thus, the phase mask is not robust to its use under an illumination at $\lambda_{B}$ which is $155 \mathrm{~nm}(25 \%)$ lower than $\lambda_{0}$. Nevertheless, the image quality seems to be improved on the focused zones of the image (links in focus and wool threads at the top and bottom of the image). This can be explained by observing the CTF at the two frequencies $15 \mathrm{lp} . \mathrm{mm}^{-1}$ and $40 \mathrm{lp} \cdot \mathrm{mm}^{-1}$ on Figure 5(c) and (d). The level of the CTF at the focused plane is twice the one of the CTF for the phase mask used at the nominal wavelength $\lambda_{0}$. This means that the contrast is too high after deconvolution. As the human eye prefers strong contrasts, the focused zones appear very sharp with the illumination at $\lambda_{B}$.

This study on a real scene image confirms the results obtained by simulation and experiments on the DoF target: a binary phase mask can reach the DoF for which it has been optimized if it is used at the nominal wavelength of optimization. If the illumination departs from the red nominal, the image quality decreases but a significant DoF extension first remains up to green wavelengths $(-16 \%)$ before to drop for blue wavelengths $(-25 \%)$. The experiments are in good agreement with theoretical simulations.

\section{CONCLUSION}

In this paper, we have studied the actual behavior of co-optimized hybrid systems for DoF extension under different monochromatic illuminations. In all investigated cases, the experiments validate the simulated behavior of the systems. As expected, the DoF reachable with an illumination at nominal wavelength is large and reaches the optimization target. If the wavelength of the monochromatic illumination deviates from the nominal one, the image quality first slightly decreases within a mainly unchanged DoF range. However, if the wavelength change becomes too strong, the DoF extension may significantly drop down up to a factor 2 . Therefore, even though attention has to be paid to avoid using the hybrid system with a too different wavelength than the design one, the DoF extension property has been proof to be somewhat robust to wavelength changes.

To go further than this present work, which considered narrow spectrum illumination, it could be interesting to study optimization and behavior of such DoF extended co-optimized hybrid systems within a more general panchromatic imaging configuration with wide spectrum illumination. It may be worth also investigating robustness to optical aberrations and to spatial undersampling.

\section{REFERENCES}

[1] E. R. Dowski et W. T. Cathey, «Extended depth of field through wave-front coding,» Appl. Opt., vol. 34, pp. 18591866, 41995. 
[2] W. T. Cathey et E. R. Dowski, «New paradigm for imaging systems,» Applied optics, vol. 41, pp. 6080-6092, 2002.

[3] B. Milgrom, N. Konforti, M. A. Golub et E. Marom, «Novel approach for extending the depth of field of Barcode decoders by using RGB channels of information,» Opt. Express, vol. 18, pp. 17027-17039, 82010.

[4] F. Diaz, M.-S. L. Lee, X. Rejeaunier, G. Lehoucq, F. Goudail, B. Loiseaux, S. Bansropun, J. Rollin, E. Debes et P. Mils, «Real-time increase in depth of field of an uncooled thermal camera using several phase-mask technologies,» Opt. Lett., vol. 36, pp. 418-420, 22011.

[5] M.-A. Burcklen, F. Diaz, F. Lepretre, J. Rollin, A. Delboulbé, M.-S. L. Lee, B. Loiseaux, A. Koudoli, S. Denel, P. Millet, F. Duhem, F. Lemonnier, H. Sauer et F. Goudail, «Experimental Demonstration of Extended Depth-of-Field F/1.2 Visible High Definition Camera with Jointly Optimized Phase Mask and Real-Time Digital Processing,» Journal of the European Optical Society, vol. 10, pp. 150461-150466, 102015.

[6] S. Elmalem, R. Giryes et E. Marom, «Learned phase coded aperture for the benefit of depth of field extension,» $O p t$. Express, vol. 26, pp. 15316-15331, 62018.

[7] F. Diaz, F. Goudail, B. Loiseaux et J.-P. Huignard, «Design of a complex filter for depth of focus extension,» Opt. Lett., vol. 34, pp. 1171-1173, 42009.

[8] R. Falcón, F. Goudail, C. Kulcsár et H. Sauer, «Performance limits of binary annular phase masks codesigned for depth-of-field extension,» Optical Engineering, vol. 56, p. 065104, 2017.

[9] A. Fontbonne, H. Sauer, C. Kulcsár, A.-L. Coutrot et F. Goudail, «Experimental validation of hybrid optical-digital imaging system for extended depth-of-field based on co-optimized binary phase masks,» Optical Engineering, vol. 58, p. 1, 112019.

[10] Y. Shi et R. Eberhart, «A modified particle swarm optimizer,» pp. 69-73, 51998. 